\title{
Revisiting the 'Venturi effect' in passage ventilation between two non-parallel buildings
}

Article

Accepted Version

Li, B., Luo, Z., Sandberg, M. and Liu, J. (2015) Revisiting the 'Venturi effect' in passage ventilation between two non-parallel buildings. Building and Environment, 92 (2). pp. 714-722. ISSN 0360-1323 doi:

https://doi.org/10.1016/j.buildenv.2015.10.023 Available at https://centaur.reading.ac.uk/45708/

It is advisable to refer to the publisher's version if you intend to cite from the work. See Guidance on citing.

To link to this article DOI: http://dx.doi.org/10.1016/j.buildenv.2015.10.023

Publisher: Elsevier

All outputs in CentAUR are protected by Intellectual Property Rights law, including copyright law. Copyright and IPR is retained by the creators or other copyright holders. Terms and conditions for use of this material are defined in the End User Agreement.

www.reading.ac.uk/centaur 
Central Archive at the University of Reading

Reading's research outputs online 
Revisiting the 'Venturi effect' in passage ventilation between two non-parallel buildings

Biao $\mathrm{Li}^{1,2}$, Zhiwen Luo, ${ }^{2, *}$, Mats Sandberg ${ }^{3}$, Jing Liu ${ }^{1,4}$

${ }^{1}$ School of Municipal and Environmental Engineering, Harbin Institute of Technology, China

${ }^{2}$ School of Built Environment, University of Reading, Reading, United Kingdom

${ }^{3}$ KTH Research School, University of Gävle, Sweden

${ }^{4}$ State Key Laboratory of Urban Water Resource and Environment, Harbin Institute of Technology, China

*Corresponding Email: z.luo@reading.ac.uk

Keywords: Ventilation capacity, urban environment, non-parallel passage, Venturi effect, pedestrian wind

This manuscript includes 12 figures and 0 tables. 
Abstract: A recent study conducted by Blocken et al. (Numerical study on the existence of the Venturi effect in passages between perpendicular buildings. Journal of Engineering Mechanics, 2008,134: 1021-1028) challenged the popular view of the existence of the 'Venturi effect' in building passages as the wind is exposed to an open boundary. The present research extends the work of Blocken et al. (2008a) into a more general setup with the building orientation varying from $0^{\circ}$ to $180^{\circ}$ using $\mathrm{CFD}$ simulations. Our results reveal that the passage flow is mainly determined by the combination of corner streams. It is also shown that converging passages have a higher wind-blocking effect compared to diverging passages, explained by a lower wind speed and higher drag coefficient. Fluxes on the top plane of the passage volume reverse from outflow to inflow in the cases of $\alpha=135^{\circ}, 150^{\circ}$ and $165^{\circ}$. A simple mathematical expression to explain the relationship between the flux ratio and the geometric parameters has been developed to aid wind design in an urban neighborhood. In addition, a converging passage with $\alpha=15^{\circ}$ is recommended for urban wind design in cold and temperate climates since the passage flow changes smoothly and a relatively lower wind speed is expected compared with that where there are no buildings. While for the high-density urban area in (sub)tropical climates such as Hong Kong where there is a desire for more wind, a diverging passage with $\alpha=150^{\circ}$ is a better choice to promote ventilation at the pedestrian level.

Keywords: urban wind, ventilation, pedestrian wind comfort, building passage 


\section{Introduction}

The influence of buildings on the wind micro-environment has aroused increasing attention in the area of urban planning and design [1-5]. Many researchers have found that the urban open space design with long and narrow passages between slab-type buildings can lead to a significant increase in local wind speed and cause wind nuisance for pedestrians [6-11]. This phenomenon is traditionally explained as the result of the Venturi effect $[6,9]$ since the flow speed in the building passage shows an increase along with a decrease in the section area, which is thought to be analog with the Venturi phenomenon described for duct flow well documented in fluid engineering [12]. This view has been widely recognized and propagated [10, 13-16]. However, recent work conducted by Blocken et al. $[17,18]$ doubted the applicability of the Venturi effect to the urban wind environment, and attributed the increase of wind speed near ground level mainly to the wind-blocking effect rather than the Venturi effect. They argued that the passage between two buildings is open to the atmosphere at the top rather than being a closed space. Therefore, with the decrease of flow cross-section area in converging flow, the maximum flow speed along the pedestrian centerline increases but is not accompanied by an increase of flow rate. The wind speed in diverging passages is however systematically larger than that in converging passages at the pedestrian level. In addition, they considered the wind-blocking effect as the most important physical process in controlling the flow condition in a passage, which causes the slowdown of wind speed upstream of the buildings, allowing part of the air mass to flow around and over the passage rather than being forced through the narrowest passage opening. 
The pioneering work from Blocken et al. $[17,18]$ has provided new insights into the 'old' question of the Venturi effect in urban wind design, but they only examined a $45^{\circ}$ converging passage and a corresponding $135^{\circ}$ diverging passage. Wind conditions under other different arrangements of passage buildings are still unclear. Existing studies have not provided sufficient guidance or a ready-to-use tool for urban design and planning professionals to guide their design practice. In the present paper, we intend to extend the work of Blocken et al. (2008a) [17] to a full range of building arrangements with the angles varying from $0^{\circ}$ to $180^{\circ}$. Numerical simulation based on CFD is employed for a detailed investigation of the wind environment, wind speed amplification, wind flow flux and drag force in passages between two long narrow buildings with different orientations. A simple empirical formula based on the simulation database is also derived to guide urban wind design practice. The overarching aim of the current study is to offer further insights into the mechanism of wind conditions in passages.

\section{Model Setup}

The passage flow between buildings is mainly influenced by the building orientation, building arrangements, building dimensions and passage width. Blocken et al. (2008b) [18] conducted wind tunnel experiments to consider a typical passage model with two identical buildings of $100 \mathrm{~m} \times 10 \mathrm{~m} \times 30 \mathrm{~m}(L \times B \times H$, in full scale $)$ placed perpendicular to each other, which is believed to be a suitable arrangement for the occurrence of Venturi flow [6]. Two types of wind flow were considered, i.e., converging flow $\left(\alpha=45^{\circ}\right)$ and diverging flow $\left(\alpha=135^{\circ}\right)$ depending on the wind direction. Buildings orientation $(\alpha)$ is defined as the angle between the building's lengthwise edge 
and the approaching wind direction. In the present study, the same definition was adopted, and wind environment conditions were investigated by extending Blocken et al. (2008a)'s work to various building orientations $(\alpha)$ : all $\alpha<90^{\circ}$ cases are referred to as converging arrangements, while all $\alpha>$ $90^{\circ}$ cases are diverging arrangements, and $0^{\circ}$ and $90^{\circ}$ cases are parallel arrangements. Schematic representations of basic passages are shown in Fig.1, which has identical models to those in [17] and all notations are the same. The passage width is assumed to be constant as $W=30 \mathrm{~m}$ (in full scale). In total, 12 cases were considered by varying $\alpha$ from $0^{\circ}$ to $180^{\circ}$ with a step-change of $15^{\circ}\left(0^{\circ}\right.$ and $180^{\circ}$ are the same cases). The top view of the building models is shown in Fig.2. Following [17], a coordinate system called the r-coordinate system is established with its origin at $\mathrm{r}_{0}$, at the location of the small cross-section of the passage (SCSP) and the unit length $r=L / 2$. The same terminology as in [17] is applied in order to make a consistent comparison, the horizontal flux $F_{\mathrm{P}}$ goes through the upstream plane $A_{\mathrm{P}}$, the horizontal flux $F_{\mathrm{H}}$ goes through the downstream plane $A_{\mathrm{H}}$, and the vertical flux $F_{\mathrm{V}}$ goes through the horizontal plane $A_{\mathrm{V}}$ at the passage top. A positive or negative flux through a plane is determined according to the direction of the normal vector in the Fig. 1. The positive direction of the coordinate is in line with the wind flow direction (positive in the downstream direction). 
(a)

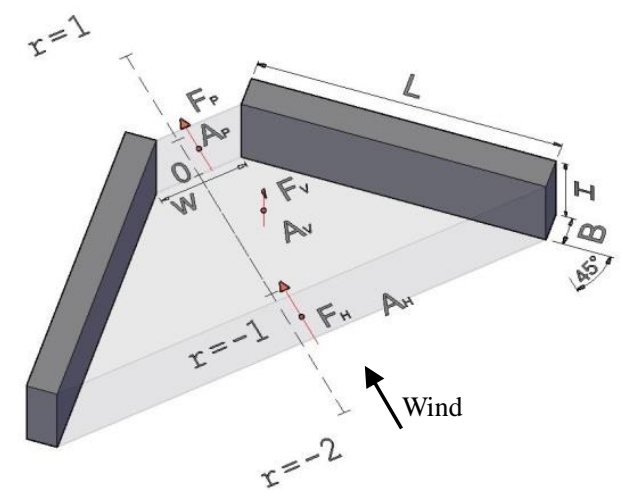

(b)

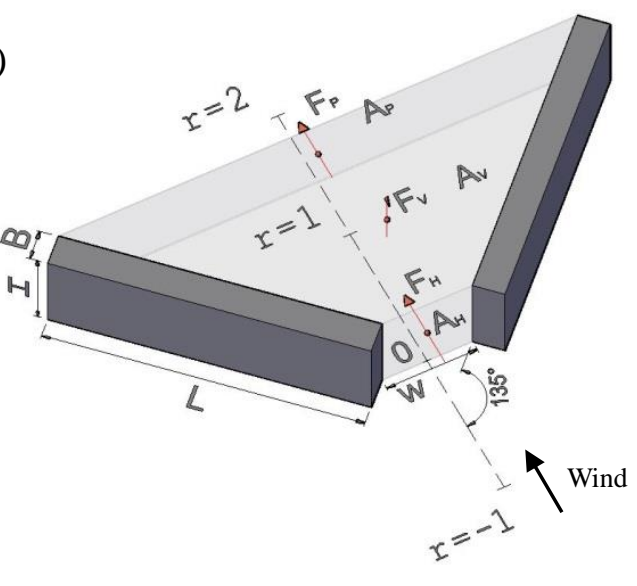

Figure 1 Illustration and notations of passages between buildings: (a) $\alpha=45^{\circ}$, convergence flow (b) $\alpha=135^{\circ}$, divergence flow

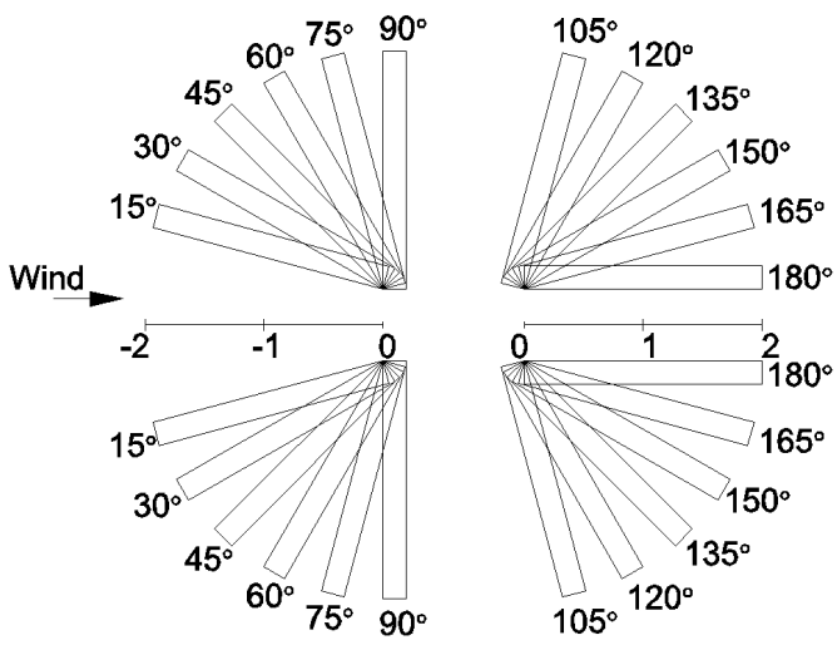

Figure 2. Top view of passages between buildings with different orientations

\subsection{CFD simulation setup}

CFD software ANSYS Fluent 14 was utilized to simulate wind flow in and around passages.

The governing equations are 3D steady-state isothermal and incompressible Reynolds-averaged Navier-Stokes equations, using the Standard $k-\varepsilon$ model as the turbulence model, which was recommended in the study [19]. In our previous study [19], we compared various $k-\varepsilon$ turbulent 
models including Standard, RNG and Realizable $k-\varepsilon$ models, and found the predicted wind speed by these models to be comparable, which agreed with other CFD studies on outdoor wind environments [20, 21]. Pressure-velocity coupling is taken care of by the SIMPLE algorithm. Pressure interpolation is second order. Second-order discretization schemes are used for both the convection terms and the viscous terms in the governing equations. A sufficiently high mesh resolution in the vertical direction within the pedestrian space [22], suitable values for $\mathrm{y}^{+}$in the passages and a Reynolds number independent flow are required in CFD simulations of the pedestrian wind environment. Therefore, our simulations were performed at the scale of 1:40 to satisfy all these requirements as in [17]. The dimensions of the computational domain are $1,400 \times 1,400 \times 400 \mathrm{~m}^{3}$ (Length $\times$ Width $\times$ Height) in full scale and buildings are placed at the center of the domain. The first-layer cells close to building surfaces are generated to ensure the y+ value falls within the range of 30-100 to satisfy the requirement of the standard wall function. In addition, the distances from the buildings to the top boundary was more than $10 \mathrm{H}$, the lateral, windward and leeward boundaries were all more than $15 \mathrm{H}$ away. The maximum blockage ratios were less than $0.6 \%$, the maximum lateral directional blockage ratio was $16.4 \%$, and all the vertical directional blockage ratios were 7.5\%, all of which show good conformation with practice guidelines [22-24]. After grid-sensitivity analysis, which was conducted on hexahedral grids including 1.2 million, 2.4 million, 3.4 million, and 9 million cells, a suitable structured hexahedral mesh with approximately 3.4 million cells was obtained. Taking the $45^{\circ}$ case as an example (Fig.3), fine grids were constructed near the building, and gradually increased toward the outer boundaries of the domain. 
(a)

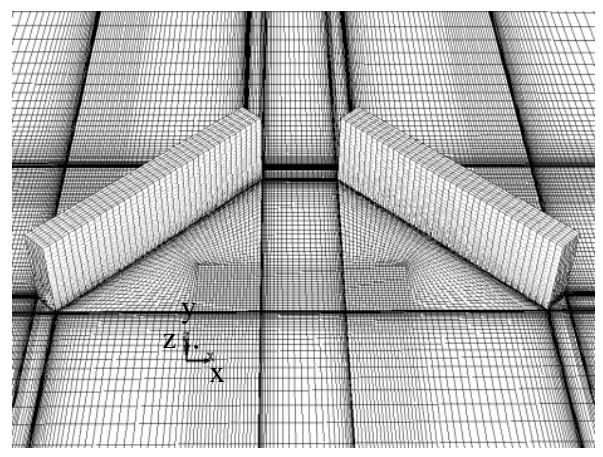

(b)

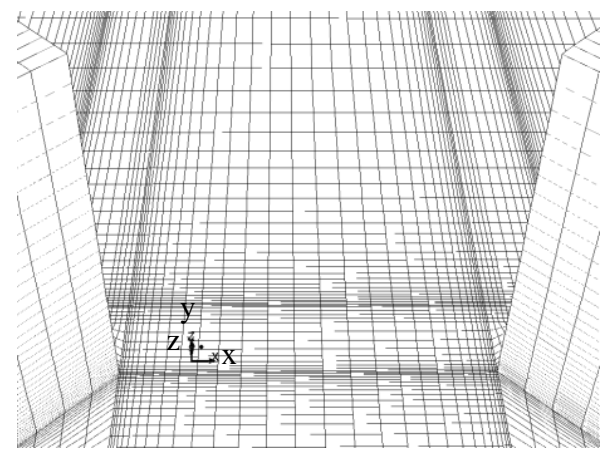

Figure 3. (a) Perspective view of the grids near and in the passage (b) Detailed view of the grids near the small cross-section of the passage (SCSP)

In these numerical simulations, following Blocken et al. (2007), a rough wall function was applied on the ground to minimize the inhomogeneity of the flow when traveling along the domain, while the sky and sides of the domain were set to be symmetry. Outflow was considered as a fully-developed, free-outflow boundary condition. Particularly, the profiles measured in the empty wind tunnel at the location where the buildings will be positioned are used as the inlet profiles in simulations with buildings, as shown in Fig.4. The inlet vertical profiles of mean wind speed, turbulence kinetic energy and turbulence dissipation rate were obtained based on wind tunnel measurements $[17,18,24]$ :

$$
\begin{gathered}
u=7.4 \times\left(\frac{z}{0.05}\right)^{0.125} \\
k=\sigma^{2}=(I U)^{2} \\
\varepsilon=\frac{u^{* 3}}{\kappa\left(z+z_{0}\right)}
\end{gathered}
$$

where $z$ is the height, $I$ is turbulence intensity. The aerodynamic roughness length $z=0.00005 \mathrm{~m}$ (CFD scale) and the friction velocity $u^{*}=0.45 \mathrm{~m} / \mathrm{s}$ were adopted from [17]. In the simulations, the 
same value for physical roughness height as for the aerodynamic roughness length is utilized. $C_{s}$ was set at its default value 0.5 and $k_{s}=0.001 \mathrm{~m}$ (CFD scale). A simulation was conducted in an empty domain to assess the extent of horizontal homogeneity as suggested by [22]. Fig.4 shows that the incident profiles along the domain are reasonably consistent with the inlet profiles, where $U_{\mathrm{g}}$ denotes the wind speed above the gradient height. The difference between mean wind speeds is trivial while the difference between turbulence intensities is comparatively significant. Overall, it presents good consistency when wind flows through the domain.

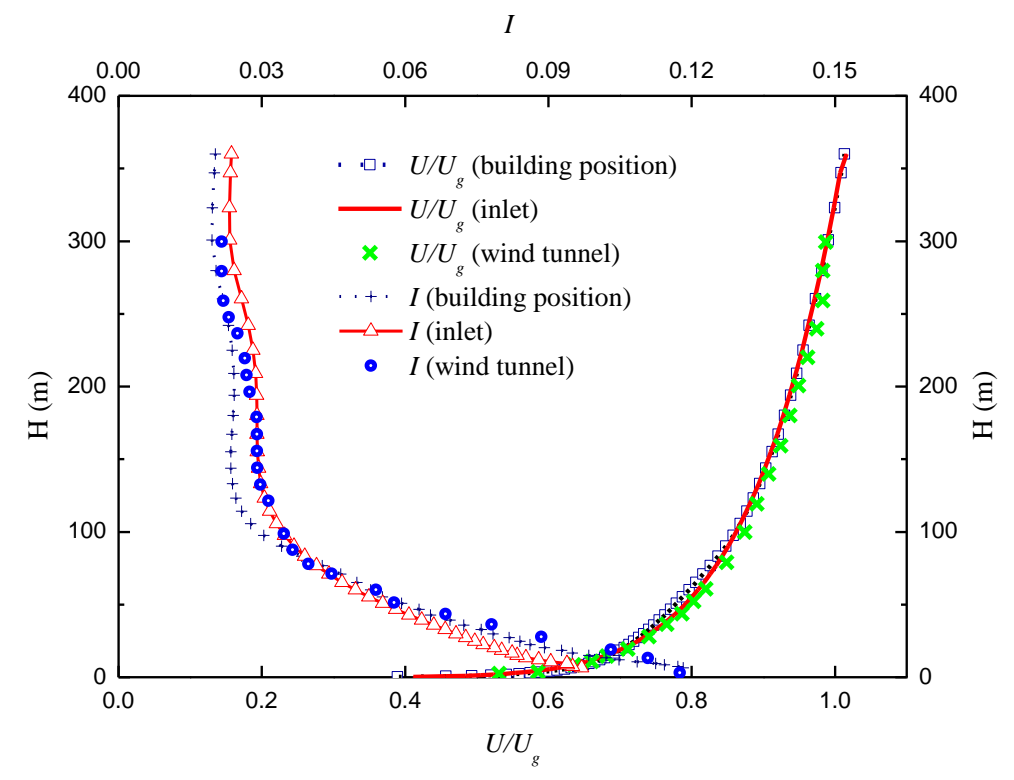

Figure 4. Vertical profiles of ratio $U / U_{g}$ and turbulence intensity $I$, measured in a wind tunnel, at the inlet and where the buildings would be positioned.

\subsection{Model Validation}

Model validation was done by comparing numerical results with wind tunnel measurements conducted by [18] at pedestrian height ( $2 \mathrm{~m}$ in real scale) along the passage center line. Wind tunnel experiments were performed at a scale of 1:400 with the reference incident wind speed of $7.4 \mathrm{~m} / \mathrm{s}$. 
Two cases were considered in the wind tunnel i.e. $\alpha=45^{\circ}$ and $135^{\circ}$ corresponding to the converging and diverging arrangements. The numerical results were presented as amplification factors for the mean wind speed. The amplification factor $K$ is defined as the ratio of $U / U_{0}$, where $U$ is local wind speed, and $U_{0}$ is reference wind speed at the height of $2 \mathrm{~m}$ (full scale) at $\mathrm{r}=0$. Fig.5 shows the comparison of the amplification factor along the passage center line $\left(K_{\mathrm{pcl}}\right)$ between experimental and simulated results. It illustrates that, generally, numerical results agree quite well with the experimental data. However, the simulation results for the converging passage are more accurate than those for the diverging passage. The largest discrepancy occurs at the outlet of the diverging flow. This may be attributable to the limitations of the RANS model in addressing the complexity of the wake effects, which has been widely acknowledged in the literature $[20,21]$. However, the current study centers its focus on the increase of the velocity where the so-called Venturi effect occurs rather than at the wake regions. This discrepancy has the minimum impact on the outcomes of the present study. It is also indicated in Fig.5 that the amplification factors $K_{\mathrm{pcl}}$ through the diverging passage is larger than that through the converging passage. In addition, the trends of $K_{\mathrm{pcl}}$ through the two types of passage are similar to each other: the peak and minimum $K_{\mathrm{pcl}}$ occur closer to $r=0.5$ and $r=-0.5$, respectively, which are both near the SCSP 
(a)

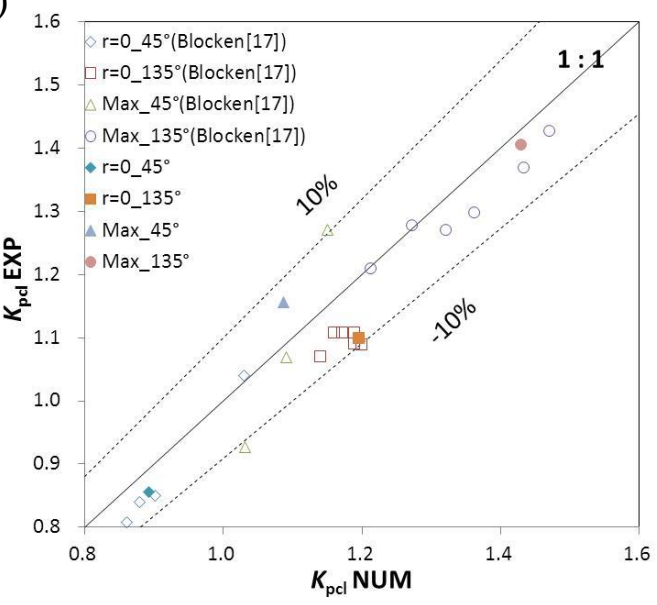

(b)

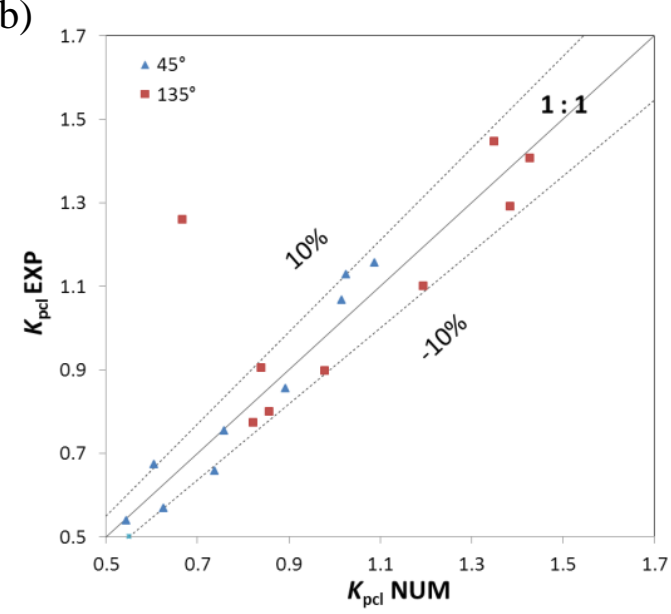

(c)

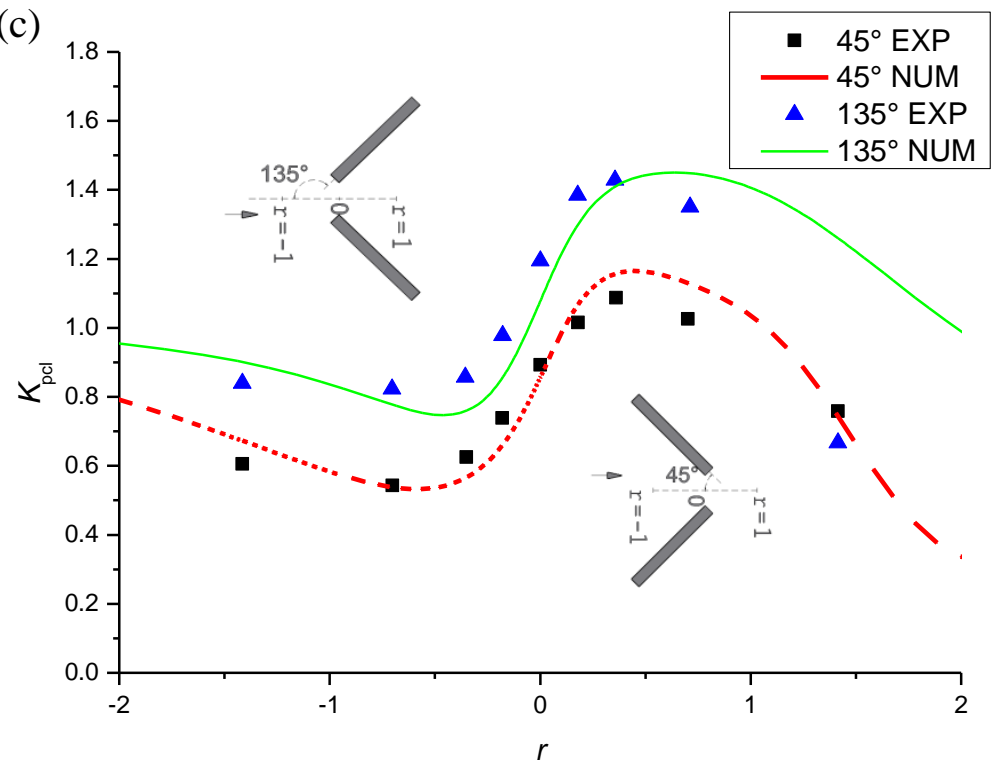

Figure 5. Comparison of experimental and simulated results: (a) $K_{\mathrm{pcl}}$ at $r=0$ and at the position where its experimental value is maximum; (b) $K_{\mathrm{pcl}}$ along the passage center line; (c) comparison between experimental data and numerical simulation resutls along the passage center line.

\section{Results and discussions}

\subsection{Converging flow vs. diverging flow}

Results are discussed in terms of the amplification factor $K$ at the height of $2 \mathrm{~m}$ (full scale). Fig.6 
shows the contours of $K$ in and around the building passage. Generally, the higher $K$-value areas appear around corner streams and within passages in all cases, and the calm-wind areas are evident near windward and leeward sides of the buildings. With the angle between the building and centerline changing to become close to $90^{\circ}$, the regions of high wind speed shrink and the regions of low wind speed expand simultaneously, corner streams become weaker and the wake area grows larger. With $\alpha$ increasing from $0^{\circ}$ to $90^{\circ}$ and then $90^{\circ}$ to $180^{\circ}$, low $K$-value regions at the windward side of buildings form near outside upstream corners at the beginning, and they grow larger and move inward to the SCSP. The largest area of windward low $K$-value regions appears near the center of buildings when $\alpha=90^{\circ}$, and then they move toward the SCSP and become smaller, until the smallest appears at $\alpha=180^{\circ}$. Therefore, the transition of low $K$-value regions to the windward of buildings is closely related to the variation of the frontal area density. 


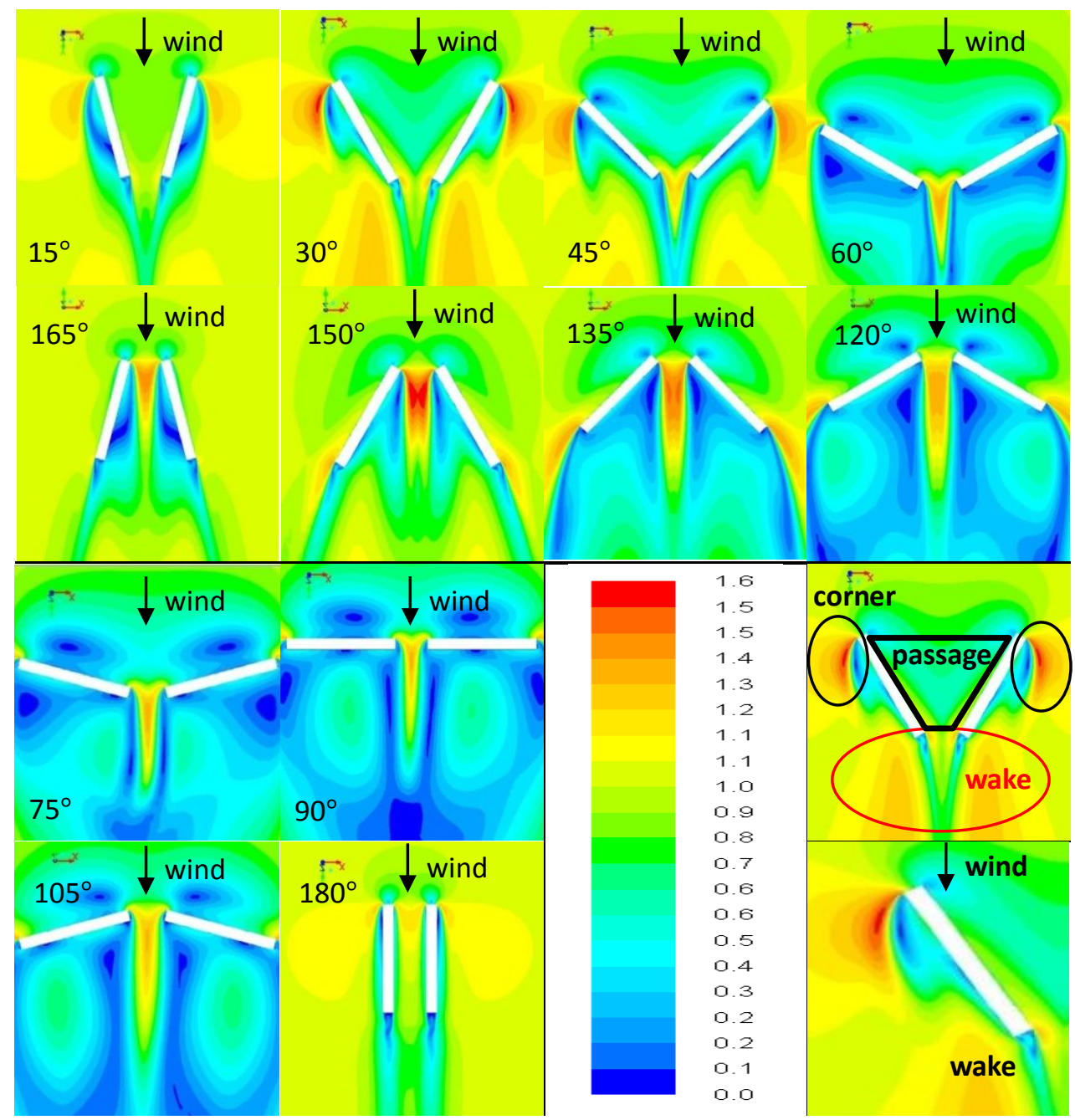

Figure 6. Amplification factor $K$ in a horizontal plane at $2 \mathrm{~m}$ height above the ground; wind direction and building orientation $\alpha$ are shown in each figure; two schematic diagrams are illustrated based on the $\alpha=30^{\circ}$ case.

It is clear that the wind speed in most diverging passages is considerably larger than that in converging counterparts. In addition, Fig. 6 shows that the $K$ value is higher near the upstream outer corners than that within the passage for all $\alpha<90^{\circ}$ cases. Similar results were observed by [17]. The greatest $K$ appears near the upstream outer corners when $\alpha<90^{\circ}$, while for $\alpha>90^{\circ}$, it locates near the opening of the passages (at upstream). Furthermore, for each group of complementary cases (the 
two cases with complementary angle $\alpha$ ), the magnitudes of high $K$-value regions are similar and all appear in upstream regions. Therefore, for passages of $\alpha>90^{\circ}$, the passage flow can be regarded as the superposition of the upstream corner streams from the two buildings; while for $\alpha<90^{\circ}$ cases, it is the superposition of the downstream corner streams. In other words, both the corner stream and the passage flow in the upstream region mostly show greater flow speeds than those in the downstream region. Moreover, [22] stated that the maximum $K$ at the outer corners $\left(K_{\mathrm{c}, \max }\right)$ is nearly independent of the passage width, and the maximum $K$ in the passage $\left(K_{\mathrm{p}, \max }\right)$ was also observed to be the same as the $K_{\mathrm{c}, \max }$ at the outer corners when the passage width is equal or wider than $30 \mathrm{~m}$. All these results show that the superposition of flows near buildings' corners plays an important role in forming the passage flow. Taking another line of argument, this explains well that wind speed in diverging passages is strikingly larger than that in corresponding converging passages, because the SCSP of diverging passages are in the upstream area where corner streams are stronger.

\subsection{Amplification factor in the passage}

Fig.7 illustrates the $K_{\mathrm{pcl}}$ as a function of $r$ along the passage center line at pedestrian height for all cases. Comparing all curves from $r=-1.5$ to 1.5 , the changing trends of $K_{\mathrm{pcl}}$ are similar but offset to each other. The following main observations can be made:

1) SCSP is very important in determining the overall characteristics of passage flow. After a general decrease of $K_{\mathrm{pcl}}$ before the SCSP, a dramatic increase would occur at the opening of the SCSP. The peak appears at approximately $0.25 r \sim 0.6 r$ downstream of the SCSP and the valleys appear at approximately $0.25 r \sim 0.5 r$ upstream of the SCSP. 
2) It is well observed that $K_{\mathrm{pcl}}$ are less than 1 for both cases when $\alpha=15^{\circ}$ and $30^{\circ}$. It demonstrates that the so-called Venturi effect is not observed in the cases of $15^{\circ}$ and $30^{\circ}$ at all, though they belong to converging flow in principle. The results support the view that the Venturi effect is not able to describe the outdoor passage flow between two symmetric buildings with an open boundary. Furthermore, the highest $K_{\mathrm{pcl}}$ occurs in the case of $150^{\circ}$, showing the wind speed mounts to 1.49 times the original wind speed when the buildings are not present. Meanwhile, the smallest $K_{\mathrm{pcl}}$ is found in the case of $\alpha=75^{\circ}$, which means this arrangement has the greatest wind-blocking effect since the wind speed in front of the passage undergoes the largest decrease, approximately 0.46 times the reference velocity. In addition, the most flat curve is evident at $\alpha=15^{\circ}$, which should be recommended as an appropriate arrangement in urban design in cold climates since the passage flow changes smoothly and exhibits lower wind speed compared with that for no buildings. While for the high-density urban area in a tropical climate, such as Hong Kong where there is a desire for more wind, the diverging passage with $\alpha=150^{\circ}$ is a better choice to promote ventilation at pedestrian levels. Therefore, these relative orientations of building blocks should be given cautious consideration during the urban design process.

3) Further delving into Figure 7, it is found that the $K_{p c l \text { min }}$ decreases from $\alpha=15^{\circ}$ to $\alpha=75^{\circ}$ and then increases for converging flows. The maximum $K_{\text {pcl-values }}\left(K_{\mathrm{pcl}, \mathrm{max}}\right)$ increases when $\alpha$ increases from $15^{\circ}$ to $90^{\circ}$. For diverging flows, $K_{p c l, m i n}$ increases from $\alpha=105^{\circ}$ to $\alpha=180^{\circ}$, and $K_{\mathrm{pcl}, \max }$ increases from $\alpha=105^{\circ}$ to $\alpha=150^{\circ}$, and then decreases from $\alpha=150^{\circ}$ to $\alpha=180^{\circ}$. 
The maximum $K_{\mathrm{pcl}, \max }$ occurs when $\alpha=150^{\circ}$ which is in accordance to the maximum ratio of flow flux penetrating into the passage through the top (shown in Fig.10).

4) Comparing extreme values in Fig.7, $K_{\text {pcl,min }}$ in each diverging case $\left(\alpha>90^{\circ}\right)$ is greater than that in the complementary converging case. In a similar manner, all $K_{\mathrm{pcl}, \mathrm{max}}$ in $\alpha>90^{\circ}$ cases are larger than their complementary converging cases except for $\alpha=105^{\circ}$, whose $K_{\mathrm{pcl}, \max }$ is smaller than that in the case of $\alpha=75^{\circ}$, also shown in the enlarged view of Fig.8. This observation adds more insights to Blocken's conclusions in [17] that wind speed for a converging passage is systematically smaller than for its complementary diverging passage as they only studied one case which had $\alpha=45^{\circ}$.

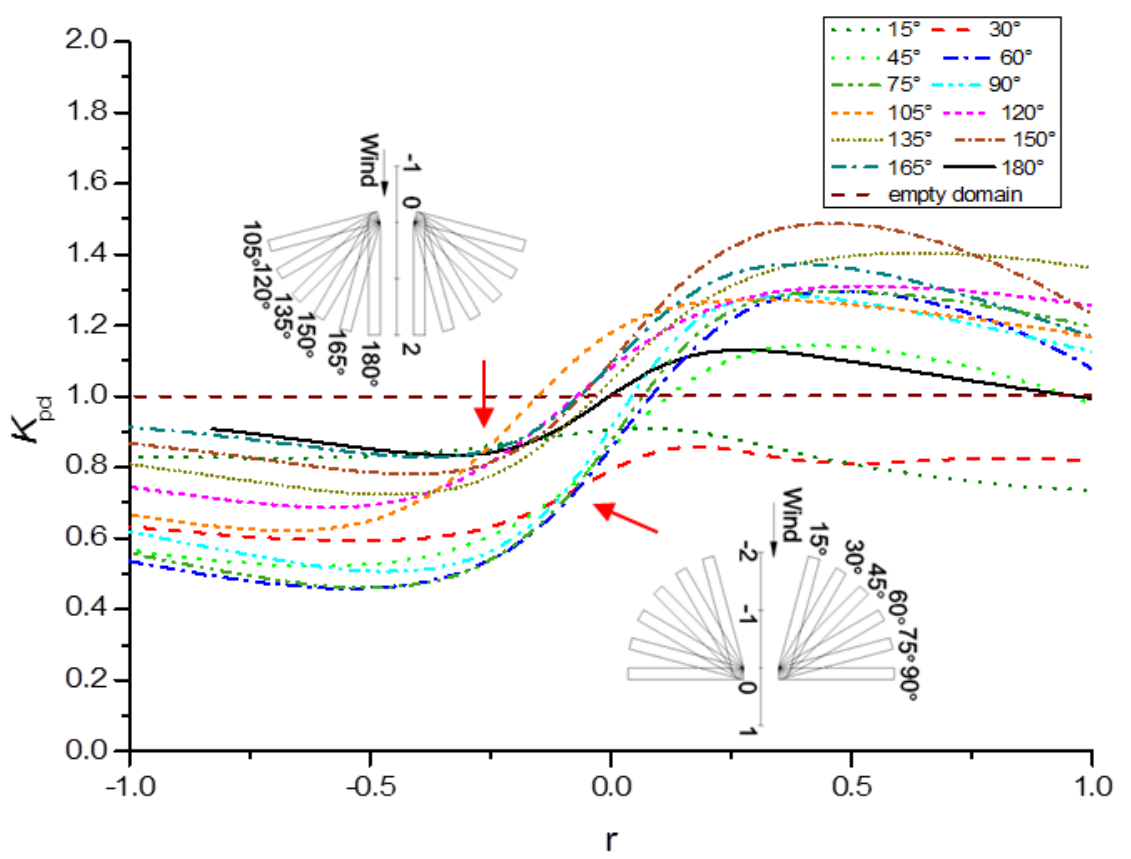

Figure 7. Amplification factor $K_{\mathrm{pcl}}$ along the passage center line 

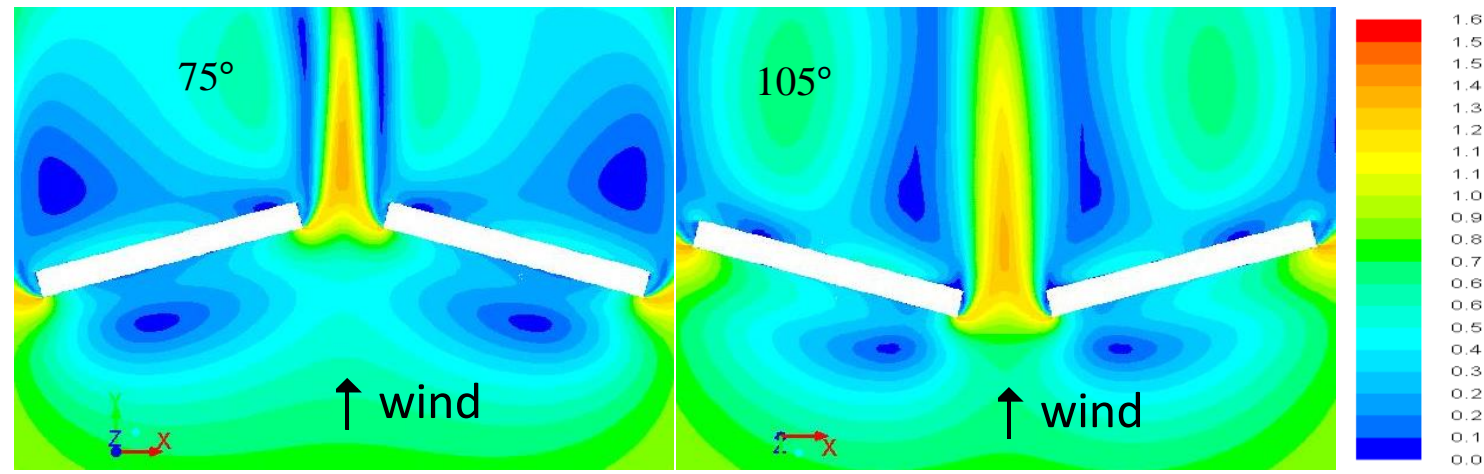

Fig. 8. Enlarged view of amplification factor $K$ in a horizontal plane at $2 \mathrm{~m}$ for the cases of $\alpha=75^{\circ}$ and $105^{\circ}$

\subsection{Drag coefficients}

A principal effect of wind flow around passage buildings is the wind-blocking effect, which forces the air to flow over and around buildings rather than just going through the narrow passage opening. Drag force/drag coefficient is normally used to describe the resistance of buildings to the air. Considering the important force of buildings acting on air, the drag coefficient, defined by Eq. (4) is exhibited in Fig.9Fig, including three drag coefficients along the $x, y, z$ coordinates (as shown in Fig.3) respectively.

$$
C_{d}=\frac{2 D}{\rho U_{g}^{2} A_{\text {total }}} \text { (4)Where } U_{\mathrm{g}} \text { is the approaching wind speed, }
$$

$A_{\text {total }}$ is the total surface area of buildings and $D$ is the total drag force of buildings. Drag coefficients for the $x$-coordinate (spanwise) are nearly zero and for the $z$-coordinate (vertical) keep at a small magnitude as around 0.05 while $\alpha$ is within $30^{\circ}$ and $150^{\circ}$, however, they constitute a significant portion of drag when $\alpha$ is near 0 and 180 although the overall magnitude is small. Moreover, different from fluxes changing dramatically in diverging passages, trends of drag 
coefficients in $y$-coordinate show a good association with the directions of building orientations, indicating the frontal area is a major factor in determining the drag force. In addition, drag coefficients for $\alpha=15^{\circ}$ and $165^{\circ}$ are approximately the same due to their equal and small frontal areas, but the drag coefficient for the converging passage is slightly larger than the corresponding diverging one in all other pairs of complementary cases. It means that the wind-blocking effect of passage buildings in these converging arrangements is more pronounced than that in corresponding diverging arrangements, which contributes more reduction of fluid speed and longer deceleration distance in converging passages as shown in Fig.6 and Fig.7. The drag coefficient is therefore the main indicator of the wind-blocking effect. Furthermore, as shown in Fig.9, the curve shape of $K_{\mathrm{pcl}, \text { min }}$ is mirrored with that of the drag coefficient, indicating the $K_{\mathrm{pcl}, \mathrm{min}}$ is inversely correlated with the drag coefficient.

a

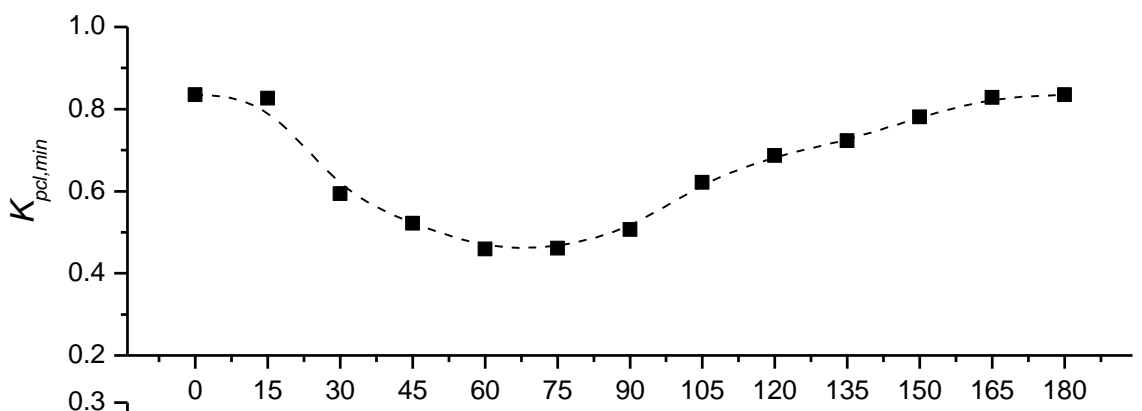

b

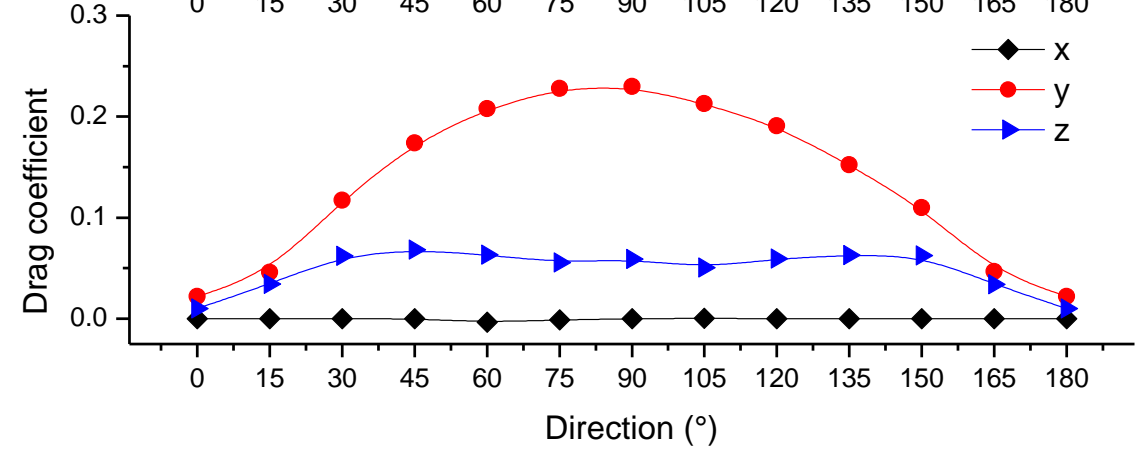

Figure 9. (a) Amplification factors as a function of the building orientations; (b) Drag coefficients 
for the $x, y, z$ coordinates defined by the $U_{\mathrm{g}}$ and the total surfaces area of buildings

\subsection{Wind flow flux}

Apart from amplification factor, flow fluxes/flow rates through the passage are also analyzed.

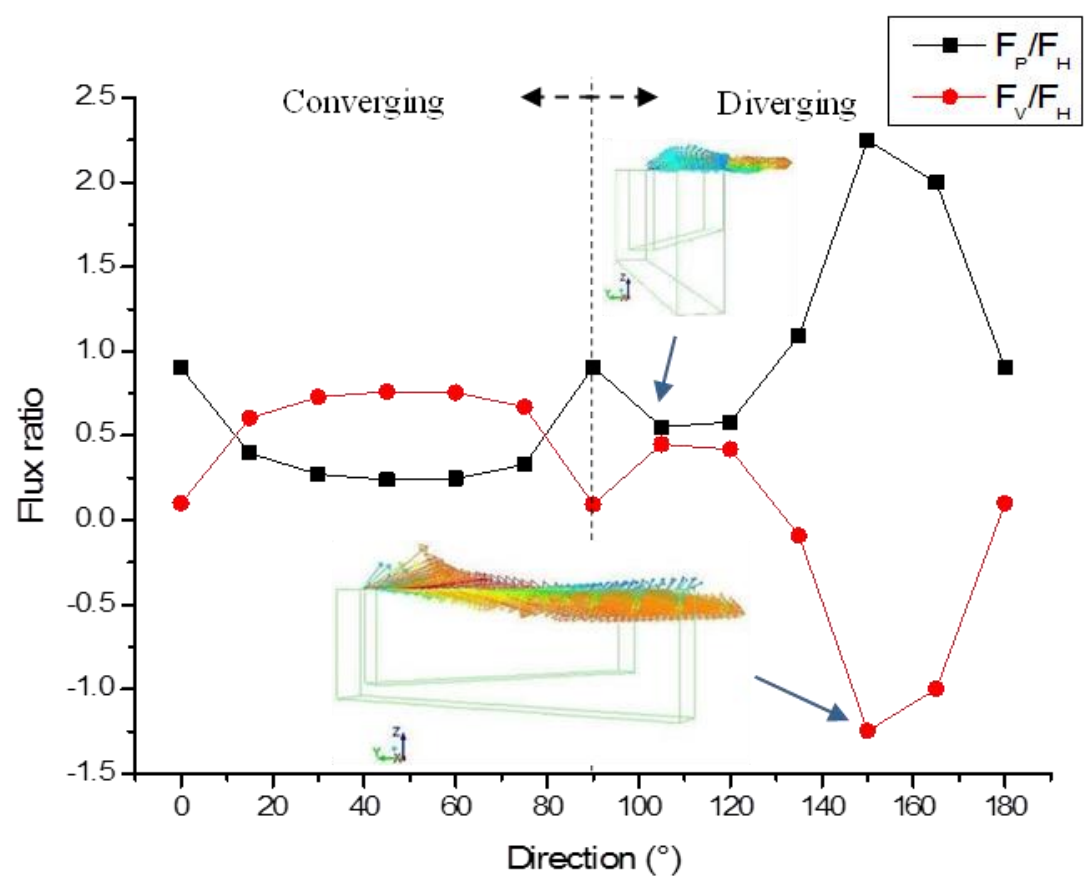

Figure 10. Flux ratios as a function of non-parallel buildings orientations (insets show vectors at $A_{\mathrm{v}}$ )

Fig.10 shows flux ratios $\left(F_{\mathrm{V}} / F_{\mathrm{H}}\right.$ and $\left.F_{\mathrm{P}} / F_{\mathrm{H}}\right)$ plotted against $\alpha$, and negative flux ratio $F_{\mathrm{V}}$ means the $A_{\mathrm{V}}$ is inlet for wind to penetrate into the passage from the top and the positive $F_{\mathrm{V}}$ represents $A_{\mathrm{V}}$ as an outlet. All converging arrangements have one inlet and two outlets, because both $F_{\mathrm{P}} / F_{\mathrm{H}}$ and $F_{\mathrm{V}} / F_{\mathrm{H}}$ are positive; the wind flows into passage volume through $A_{\mathrm{h}}$ and out through both the top $A_{\mathrm{V}}$ and the downstream $A_{\mathrm{P}}$. In contrast, diverging arrangements show more complex flow conditions. While the case of $\alpha=105^{\circ}, 120^{\circ}$ and $180^{\circ}$ are similar to converging passages with one inlet and two outlets, there are two inlets and one outlet in the cases of $\alpha=135^{\circ}, 150^{\circ}$ and $165^{\circ}$. This confirms the 
study in [17] for the diverging flow of $135^{\circ}$, and is further extended to the cases of $150^{\circ}$ and $165^{\circ}$. However, our new study reveals that not all diverging flows show a similar trend. As shown in the vector diagram in Fig.10, the reattachment flow enters the passage space from the $A_{\mathrm{V}}$ plane, causing fluxes to reverse from positive to negative in the $\alpha=135^{\circ}, 150^{\circ}$ and $165^{\circ}$ cases. In addition, as observed from Fig. 6 and Fig.10, passage areas for diverging arrangements $\alpha=105^{\circ}$ and $120^{\circ}$ are mainly located in the cavity zones of their wakes, but passage spaces in $\alpha=135^{\circ}, 150^{\circ}$ and $165^{\circ}$ cases are larger, and beyond the cavity zone, so the reattachment flow separating from the buildings' roofs will enter through the top surface $A_{\mathrm{V}}$. Therefore, the flow pattern in the diverging passage flow can be more intricate, being composed of the wind-blocking effect and the wake effect. Moreover, the passage arrangement $\alpha=180^{\circ}$ is the same as that of $\alpha=0^{\circ}$, in which the wake region locates to the leeside of buildings and downstream of the passage area, so it is more like converging passages, influenced mainly by the wind-blocking effect. 


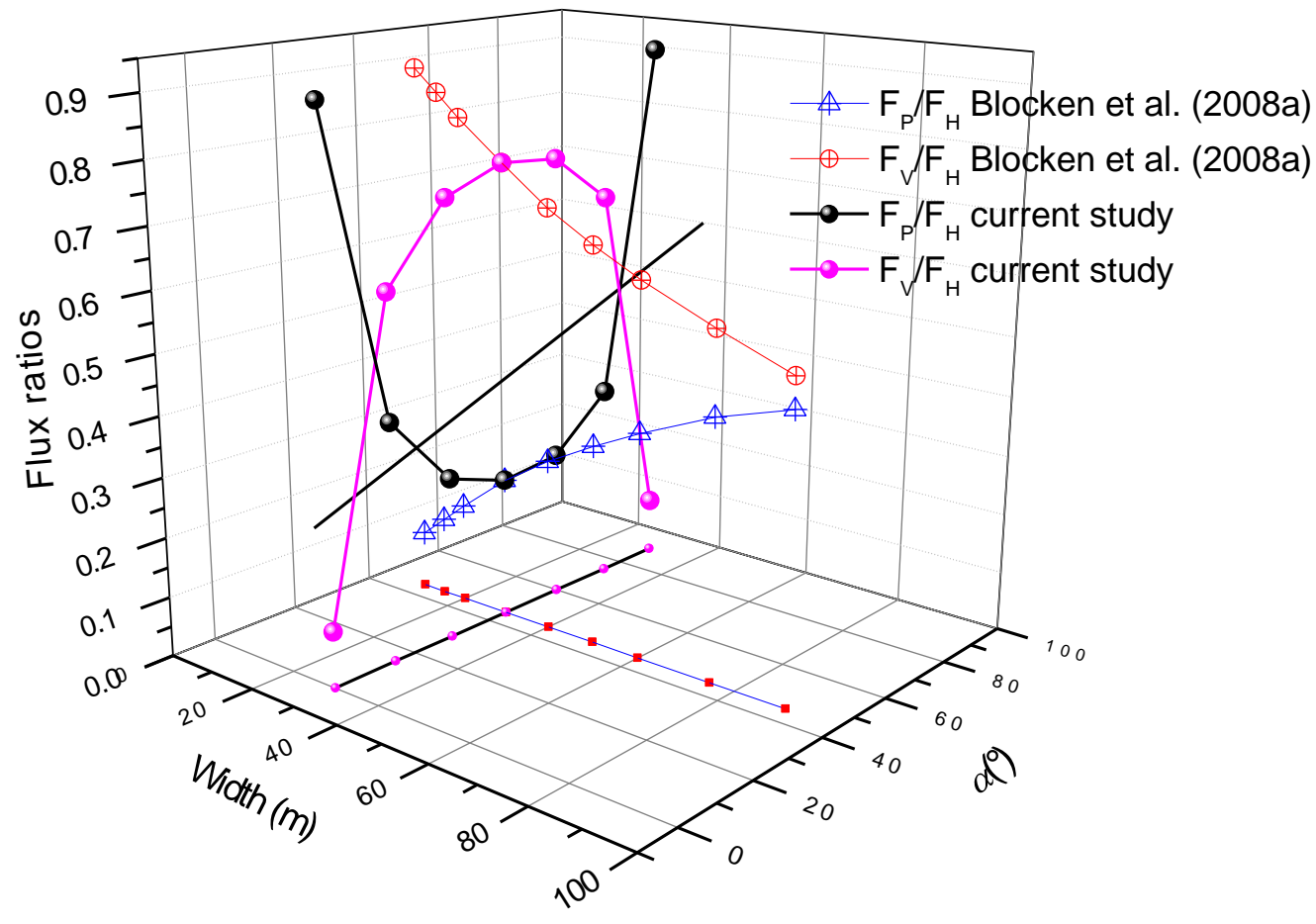

Figure 11 Flux ratios $F_{\mathrm{P}} / F_{\mathrm{H}}$ and $F_{\mathrm{V}} / F_{\mathrm{H}}$ against the passage width (converging passage $\alpha=45^{\circ}$ but different passenger widths- Blocken et al. (2008a)), and against the buildings orientation $(\alpha)$, (passage width $w=30 \mathrm{~m}$, but different orientations- current study).

The wind-blocking effect refers to the resistance of the external flow by the presence of buildings, whose morphological characteristics are essential in terms of frontal area, plan area, shape and layout. In real design practice, urban planners hope to use simple empirical formula being able to predict the resultant wind conditions based on these urban morphological parameters. We firstly combined the data from [17] for a converging flow with different passage widths and our present study of a constant passage width of $30 \mathrm{~m}$ but different building orientations in the converging flow category as depicted in Fig.11. It shows that the fluxes of converging passages are largely influenced by the wind-blocking effect characterized by the frontal area (represented by building 
orientation in the current study) and the width of the passage. Moreover, Fig.11 also demonstrates that our results have a good agreement with those of Blocken et al in [17]. Given the converging passage enclosed by two non-parallel symmetrically-placed buildings, we hypothesize that the fluxes of converging passages are mainly influenced by the width of the passage $(w)$, the length of the building $(L)$ and the building's orientation $(\alpha)$. A simple formula describing $F_{\mathrm{P}} / F_{\mathrm{H}}(\mathrm{Eq} .5)$ in relation to the above-mentioned parameters was derived by fitting the data to $C_{f l u x}$ using the algorithm of logarithm and Excel software. $C_{f l u x}$ is a flux coefficient which was created by synthesizing the morphological variables and defined in Eq.6. This resulted in constants as shown in Eq.5.

$$
\begin{gathered}
\frac{F_{\mathrm{P}}}{F_{\mathrm{H}}}=1-\frac{F_{\mathrm{V}}}{F_{\mathrm{H}}}=0.1891 \times \ln \left(C_{f l u x}\right)+0.4745 \\
C_{f l u x}=\frac{w}{c+L \sin 2 \alpha}
\end{gathered}
$$

Where $L=100 \mathrm{~m}$ is the length of the passage building; $c$ is a function of $w(10 \leq w \leq 90, \mathrm{~m})$, and follows the empirical Eq.7. $c$ was formulated by fitting to $w$ using algorithm of polynomial and Origin 8.5, and this resulted in constants as shown in Eq.7. Those constants are derived from the simulations data of [17] and the current study.

$$
c=-75.095+6.171 \times w-0.168 \times w^{2}+0.002 \times w^{3}-7.399 \times 10^{-6} \times w^{4}
$$

Fig 12 shows the results of Eq.5 with both various passage widths and building orientations, indicating the fairly good accuracy of the fitting equation. It can roughly estimate the flux ratio of passages for converging flow formed by two symmetric slab-type buildings under various buildings 
arrangements. Since the flux data used for the derivation of those parameters were only deduced from the two studies, the applicability of the equation is for $10 \leq w \leq 90$ and $0^{\circ} \leq \alpha \leq 90^{\circ}$ only. We further test the applicability of the empirical equation by investigating two extra cases ( $\mathrm{W}=50 \mathrm{~m}$ and $\alpha=60^{\circ}$ and $35^{\circ}$ ) which were not previously included in our database for deriving the equation. The CFD simulation results for the two extra cases agree very well with the prediction from the empirical equations (Eq.5-7), as shown in Figure 12. The red-filled triangle and the blue-filled star represent the two extra cases, both of them fall within the $\pm 10 \%$ performance lines.

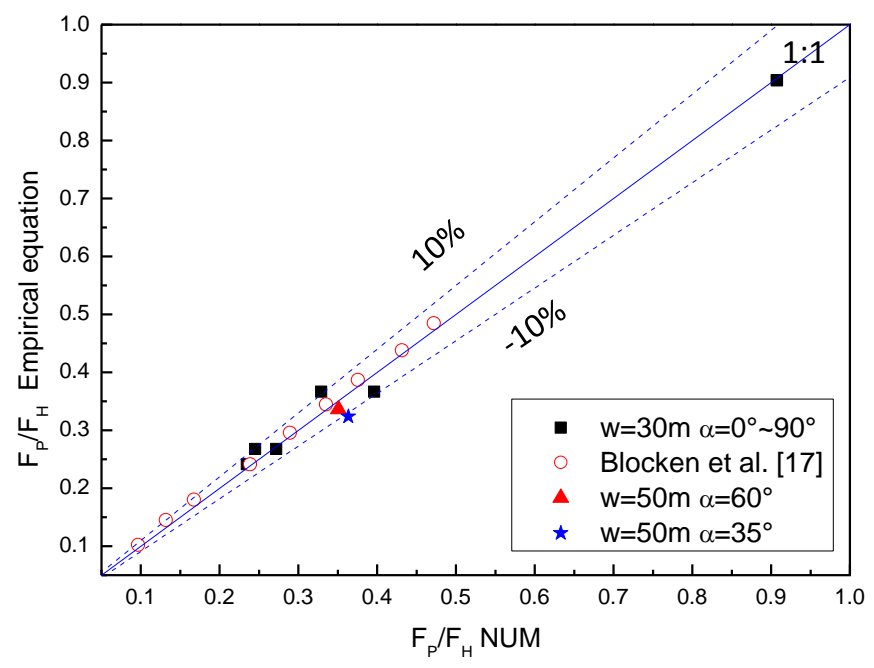

Figure 12. Fitting accuracy of flux ratios against various passage widths and buildings orientations.

\section{Conclusions}

The passage flow between two symmetric slab-type buildings with various orientations has been studied by CFD in order to provide more insight into the mechanism of wind flow in building passages. A group of simplified passage models with varying angles $\left(0^{\circ}\right.$ to $\left.180^{\circ}\right)$ between buildings and the centerline was developed by extending the previous work of [17]. Structured hexahedral grids were constructed and the horizontal homogenization and grid sensitivity were discussed. In 
addition, the numerical results were validated by corresponding wind tunnel measurements available in the literature. Wind environments, characteristics of passage flow, drag coefficients and flow fluxes of passages with different building arrangements were investigated. The following conclusions can be made:

1) The flow in diverging passages can be considered as a superposition of the corner flow near the upstream outer corners of buildings; and converging passages flow can be considered as a superposition of the downstream corner streams of buildings. Since the SCSP of diverging passages is near the upstream region, where corner streams are stronger, the wind speed with diverging passages is significantly larger than in the corresponding converging passage.

2) The trends for amplification factor $K$ for all cases are comparable; values for both peaks and valleys appear near the SCSP. In particular, in the cases of $\alpha=15^{\circ}$ and $30^{\circ}$, the wind speed at pedestrian level in the whole passage is smaller than that in the empty domain without the presence of buildings, which supports the statement that the Venturi effect is unsuitable to describe the outdoor passage flow with open boundaries. In addition, all converging passages have longer deceleration distances and smaller $K_{p c l, m i n}$ than diverging passages, because converging passages have more significant wind-blocking effects compared to complementary diverging passages. This is also reflected in drag coefficient analyses.

3) Fluxes reversed from outflow to inflow on the $A$ v planes in the cases of $\alpha=135^{\circ}, 150^{\circ}$ and 
$165^{\circ}$, indicating that the flow pattern in diverging passages flow can be more complex. Within the passages flow, the wind-blocking effect is the major factor upstream of the SCSP. For the converging passage flow between two symmetric slab-type buildings, a simple prediction model for the flux ratio of the passage flow is derived as a function of the flux coefficient $C_{f l u x}$, which is defined by the passage width and the buildings' orientations. Moreover, the trends of drag coefficients for the $y$-coordinate show good associations with the directions for building orientations.

4) A passage with $\alpha=75^{\circ}$ has the greatest wind-blocking effect since the wind speed in the passage has a decrease of approximately 0.55 times. Passages with $\alpha=15^{\circ}$ are highly recommended for urban neighborhood design in cold and temperate climates since the passage flow changes smoothly and a relative lower wind speed is expected compared to that with no buildings. Meanwhile, for the high-density urban areas in a (sub)tropical climate, such as Hong Kong where there is a desire of more wind, the diverging passage with $\alpha=150^{\circ}$ is a better choice to promote ventilation at the pedestrian level.

\section{Acknowledgement}

This work was inspired by the exciting lecture from Prof. Bert Blocken when the corresponding author ZL attended the Urban Physics School in Cyprus in 2012. ZL would also like to thank the ERASMUS mobility scheme to support the academic exchange with the University of Gävle, in Sweden. The project was financially supported by the Walker Institute Fund from the University of 
Reading.

\section{References}

[1] Lee R.X., Jusuf S.K., Wong N.H. The study of height variation on outdoor ventilation for Singapore's high-rise residential housing estates. International Journal of Low-Carbon Technologies, 2013(0):1-19.

[2] Ng E. Policies and technical guidelines for urban planning of high-density cities - air ventilation assessment (AVA) of Hong Kong. Building and Environment, 2009,44(7):1478-1488.

[3] Tominaga Y., Mochida A., Shirasawa T. et al. Cross comparisons of CFD results of wind environment at pedestrian level around a high-rise building and within a building complex. Journal of Asian Architecture and Building Engineering, 2004,3(1):63-70.

[4] Stathopoulos T., Wu H., Bédard C. Wind environment around buildings: a knowledge-based approach. Journal of Wind Engineering and Industrial Aerodynamics, 1992,44(1):2377-2388.

[5] Moonen P., Defraeye T., Dorer V. et al. Urban physics: effect of the microclimate on comfort, health and energy demand. Frontiers of Architectural Research, 2012,1(3):197.

[6] Gandemer J. Wind environment around buildings: aerodynamic concepts. Proceedings of the 4th International Conference on Wind Effects on Buildings and Structures, Heathrow,1975:423-432.

[7] Wiren B. A wind tunnel study of wind velocities in passages between and through buildings. Proceedings of the 4th International Conference on Wind Effects on Buildings and Structures, (Heathrow 1975),1975:465-475.

[8] Stathopoulos T., Storms R. Wind environmental conditions in passages between buildings. 
Journal of Wind Engineering and Industrial Aerodynamics, 1986,24(1):19-31.

[9] Lawson T.V. Wind effects on buildings: design applications . Spon Press,1980.

[10] Dutt A. Wind flow in an urban environment. Environmental Monitoring and Assessment, 1991,19(1):495-506.

[11] Blocken B., Carmeliet J. Pedestrian wind environment around buildings: Literature review and practical examples. Journal of Thermal Envelope and Building Science, 2004,28(2):107-159.

[12] Venturi G.B. Experimental enquiries concerning the principle of the lateral communication of motion in fluids: Applied to the explanation of various hydraulic phenomena. Translated from the French by W. Nicholson, 1st English Ed., J. Taylor, Architectural Library, High-Holborn, London:1799.

[13] Klemm K., Jablonski M. Wind speed at pedestrian level in a residential building complex.The 21th Conference on Passive and Low Energy Architecture, 2004:19-22.

[14] Reiter S. Assessing wind comfort in urban planning. Environment and Planning. B, Planning \& Design, 2010,37(5):857.

[15] Kubota M.T., Ahmad S. Measurement of the wind flow in residential areas of Johor Bahru Metropolitan City toward planning guidelines for energy saving city in Malaysia. Simposium Kebangsaan Masyarakat Bandar,2004:1-12.

[16] Asfour O.S. Prediction of wind environment in different grouping patterns of housing blocks. Energy and Buildings, 2010,42(11):2061-2069.

[17] Blocken B., Moonen P., Stathopoulos T. et al. Numerical Study on the Existence of the 
Venturi Effect in Passages Between Perpendicular Buildings. Journal of Engineering Mechanics, 2008,134(12):1021-1028.

[18] Blocken B., Stathopoulos T., Carmeliet J. Wind environmental conditions in passages between two long narrow perpendicular buildings. Journal of Aerospace Engineering, 2008,21(4):280-287.

[19] Hang J., Luo Z., Sandberg M., et al. Natural ventilation assessment in typical open and semi-open urban environments under various wind directions. Building and Environment, 2013,70:318-333.

[20] Mochida A., Tominaga Y., Murakami A. et al. Comparison of various k- $\varepsilon$ models and DSM applied to flow around a high-rise building - Report on AIJ cooperative project for CFD prediction of wind environment. Wind and Structures, 2002,5: 227-244

[21] Tominaga Y., Mochida A., Murakami A. and Sawaki S. Comparison of various revised k-e models and LES applied to flow around a high-rise building model with 1:1:2 shape placed within the surface boundary layer. Journal of Wind Engineering and Industrial Aerodynamics, 2008, 96 (4): $389-411$

[22] Blocken B, Carmeliet J, Stathopoulos T. CFD evaluation of wind speed conditions in passages Between Parallel Buildings_ _effect of Wall-function Roughness Modifications for the Atmospheric Boundary Layer Flow. Journal of Wind Engineering and Industrial Aerodynamics, 2007,95 (9):941-962.

[23] Blocken B. Computational Fluid Dynamics for urban physics: Importance, scales, possibilities, limitations and ten tips and tricks towards accurate and reliable simulations. Build Environ, 
2015;91:219-45.

[24] Richards P, Hoxey R. Appropriate boundary conditions for computational wind engineering models using the K-€ turbulence model. Journal of Wind Engineering and Industrial Aerodynamics, 1993, 46:145-153. 